\title{
Article \\ Spintronic RF-Direct on-off Keying Modulation Using a Frequency Division Multiplex
}

\author{
Inn-Yeal Oh *, Min-Soo Kang, Kun-Sik Kim and Chang-Ha Choi * \\ Division of Smart Automotive Engineering, Sunmoon University, Asan Chungnam 31460, Korea; \\ kang10101@sunmoon.ac.kr (M.-S.K.); smartkskim@sunmoon.ac.kr (K.-S.K.) \\ * Correspondence: innyealoh@sunmoon.ac.kr (I.-Y.O.); chchoi@sunmoon.ac.kr (C.-H.C.)
}

Citation: Oh, I.-Y.; Kang, M.-S.; Kim, K.-S.; Choi, C.-H. Spintronic RF-Direct on-off Keying Modulation Using a Frequency Division Multiplex. Electronics 2021, 10, 2200. https://doi.org/10.3390/ electronics 10182200

Academic Editor: Tadeusz A. Wysocki

Received: 22 July 2021

Accepted: 6 September 2021

Published: 9 September 2021

Publisher's Note: MDPI stays neutral with regard to jurisdictional claims in published maps and institutional affiliations.

Copyright: (c) 2021 by the authors. Licensee MDPI, Basel, Switzerland. This article is an open access article distributed under the terms and conditions of the Creative Commons Attribution (CC BY) license (https:// creativecommons.org/licenses/by/ $4.0 /)$.

\begin{abstract}
This paper presents the results of spintronic wireless communication using a new modulation method, spin RF-direct on-off keying modulation using a frequency division multiplex (FDM), which modulates amplitude and frequency simultaneously with a spin torque nano-oscillator (STNO) array. For the new modulation technique, each channel can be assigned to every STNO on the STNO array, and each STNO is simultaneously modulated by on-off keying with digital data directly. These modulation technologies have the advantage of increasing the data transmission rate by the number of operating STNOs. In recent years, efforts to increase the speed of transmission are important as the amount of data it sends increase. The receiver demodulates data on every channel through a band pass filter acquiring the frequency assigned to each channel, respectively. To initially confirm the possibility of realizing spin RF-direct on-off keying modulation using an FDM, we demonstrated the proposed modulation technique with two STNOs in an array, setting one STNO at $3.5 \mathrm{GHz}$ frequency and the other at $4.2 \mathrm{GHz}$, and communicated wirelessly with a two-channel receiver in the near field. A data rate of up to $4 \mathrm{Mbps}$ is obtained with the new modulation technique under $10 \mathrm{~mm}$ distance, and the DC power consumption is $0.18 \mathrm{~mW}$ per STNO in transmitter, including logic circuit operation.
\end{abstract}

Keywords: dual channel; frequency division; multiplex; spintronic oscillator; STNO; array

\section{Introduction}

As the use of multimedia based on AI increases in IoT environment, the need to increase transmission speed is rising. In addition, devices used in IoT environments often operate based on battery, so low-power consumption is important, and small-sized implementations are required to be applied anywhere. In this environment, communication technology based on a spin torque nano-oscillator (STNO) technology is becoming an alternative.

An STNO features an ultra-wide band-tuning range that exceeds nearly $100 \%$, and it has the advantage of being driven by low power consumption operations $[1,2]$.

Furthermore, an STNO has a remarkably small size (100 $\mathrm{nm}$ or less in diameter) as the unit of STNO itself has an oscillation microwave frequency. An STNO can thus easily be structured with an array, as STNOs are made hundreds of nanometers in size.

Owing to these characteristics of STNO, it has been reported that an STNO can feasibly be used for wireless communication by using frequency shift keying (FSK) or phase shift keying (PSK) modulation [3-8]. However, although the transmission rate is announced up to $4 \mathrm{Mbps}$, it has limitations in improving the transmission rate due to the poor frequency oscillation characteristics, and frequency nonlinearity characteristics of STNO's current.

A new modulation method is required for the realization of adequate communication using an STNO. We use on-off keying (OOK) modulation $[9,10]$. This non-coherent communication method for wireless transmission allows data transmission via air without changing the frequency to transmit wirelessly, even in the presence of STNO non-linearity 
phenomena. Additionally, as STNO has the setting time in nano-second units of fast oscillation signals [11], it has the advantage of realizing high speed transmission through OOK operation.

By exploiting nanotechnology, spintronic devices can be easily realized in an STNO array structure, and each STNO can be set to one of the isolated various frequencies among many channels that can be assigned due to wideband operation. Based on this, data are carried at each frequency for every STNO.

We then propose a new modulation concept that is adaptable to the STNO in the study, and provides frequency and amplitude modulation simultaneously. It facilitates spintronic wireless communication at higher data rates according to the number of STNOs within an array, as OOK is executed with each frequency assigned at each STNO with the targeted frequency. We have previously reported on the need for a new communication concept that can be adapted to STNO characteristics, as well as the result of a modulation technique, in [10]. In this manuscript, we report spintronic communication results between a new spintronic modulator and demodulator developed by the authors as well as a transmitter and receiver considering communication link budgets in a new communications system with spintronic oscillators. Communication performance is also analyzed describing transient characteristics with the Landau-Lifshitz-Gilbert-Slonczewski (LLGS) equation.

\section{Wireless Spintronic Communication}

The spin transfer torques of a free ferromagnetic layer are described in terms of magnetization dynamics, $\mathrm{M}(\mathrm{r}, \mathrm{t})$, and can be derived by the Landau-Lifshitz-Gilbert (LLG) equation which calculates the precession of the spin-polarized magnetization from dipolar interactions and damping [12,13].

The STNO is operated through the spin transfer torques in a spin nano-pillar by flowing the current across a spin nano-pillar, and/or applying a magnetic field around a spin nano-pillar, which is comprised of three layers: the free ferromagnetic layer, the spacer, and the pinned ferromagnetic layer.

The current passing through the polarized magnetization can generate a spin wave signal with angular momentum in the free layer caused from an effective negative damping.

For the wireless communication applying an STNO, a spin torque magnetic tunnel junction device is fabricated. Figure 1 shows a block diagram of a fabricated STNO structure, and the measurement setup.

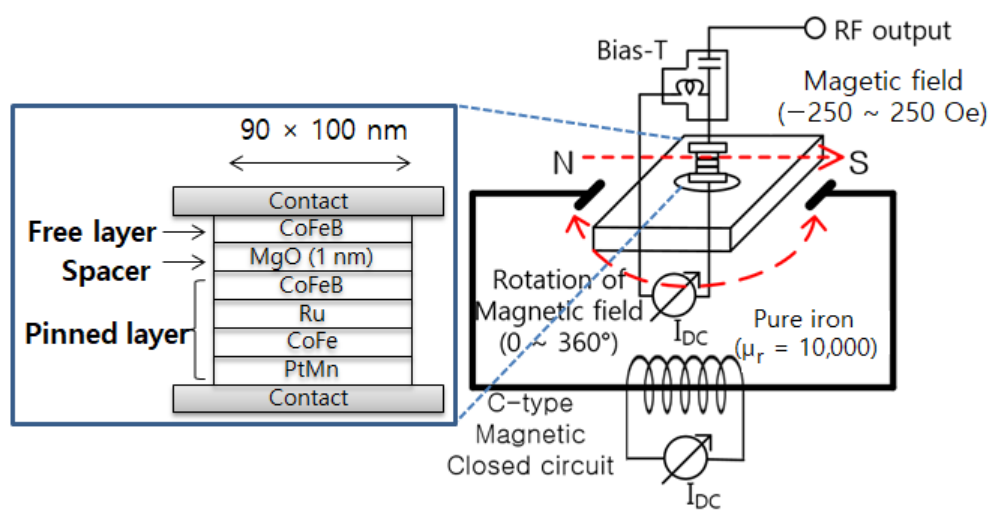

Figure 1. A block diagram of a fabricated STNO structure, and the measurement setup.

A stacking structure of $\mathrm{Co}_{6} \mathrm{Fe}_{2} \mathrm{~B}_{2}(2 \mathrm{~nm}$ depth) is deposited on the synthetic structure pinned layer by a magnetron sputtering process as a free layer. The pinned and free layers are separated by a thin $\mathrm{MgO}$ (1 nm depth) layer as a tunnel barrier. All of the stacked layers are manufactured by a single deposition process. After an e-beam masking process, ion milling etching process is carried out, and the etch is stopped in the middle of the $\mathrm{CoFeB}$ pinned layer. At that time, the junction size is decided as $90 \times 100 \mathrm{~nm}^{2}$. A post annealing process is carried out over $30 \mathrm{~min}$ at $300{ }^{\circ} \mathrm{C}$, under a $4 \mathrm{kOe}$ magnetic field. 
For measuring an STNO, a C-type magnetic closed circuit was placed at the center of the probe station for a function of the applied magnetic field. A constant current was applied to the electromagnet and a fringing field was generated at the core gap. The fabricated magnetic tunnel junction (MTJ) was located at the center of the core gap. Thus, magnetic flux line is aligned in parallel to the wafer plane. This magnetic flux line is also mechanically rotated from 0 to 360 degrees.

The magnetoresistance (MR) of MTJ device has a dependence of the applied magnetic field. When the magnetic field is applied, the MR is appeared into one of two states, anti-parallel (AP) or parallel (P) [14]. The MR of the fabricated MTJ between AP and P relation of magnetization was found to be from $48 \Omega$ to $80 \Omega$ by a magnetic field from -250 Oe to 250 Oe.

Figure 2a shows the fabricated STNO oscillation characteristics of frequency depending on the magnetic field. Frequency variation is from $2.1 \mathrm{GHz}$ to $5.0 \mathrm{GHz}$ when an external magnetic field is applied from 0 Oe to 350 Oe. The STNO frequency tuning ratio is around $80 \%$, whereas the LC-VCO electrical circuit generally has difficulty obtaining a tuning range above $20 \%$. Figure $2 \mathrm{~b}$ shows the oscillation frequency can also be set by the rotation field angle at the one magnetic field condition. Figure 2 notices that if the frequency is set into $3.5 \mathrm{GHz}$ and $4.2 \mathrm{GHz}$, the magnetic field could be adjusted into the $125 \mathrm{Oe}$ and $175 \mathrm{Oe}$ at the same rotation field angle, or the rotation field angle be into 0 degrees and 45 degrees at the 175 Oe same magnetic field.

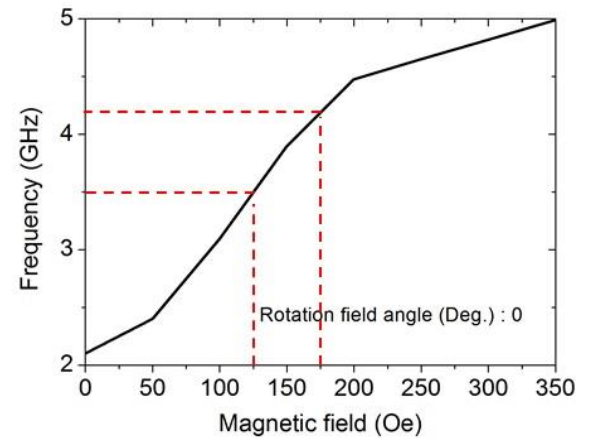

(a)

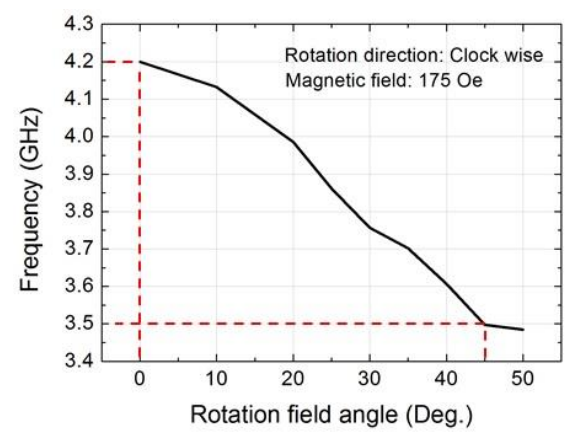

(b)

Figure 2. Spintronic oscillation characteristics of the fabricated STNO depending on (a) the magnetic field and (b) the rotation field angle at the condition of IDC $=1.5 \mathrm{~mA}$. (1 Oe (Oersted) $=1$ Gauss in air $=0.1$ millitesla).

Figure 3 shows that the peak power level and signal linewidth vary between $-71 \mathrm{dBm}$ and $-76 \mathrm{dBm}$ and $130 \mathrm{MHz}$ and $260 \mathrm{MHz}$, respectively, when an external magnetic field is applied from 0 Oe to $300 \mathrm{Oe}$ at the condition of a zero-rotation field angle, and a $1.5 \mathrm{~mA}$ current DC supply flowing through the STNO. In particular, $130 \mathrm{MHz}$ linewidth measured in our fabricated STNO is meaning the phase noise is $-63 \mathrm{dBc}$ at an offset of $130 \mathrm{MHz}$. Figure 3 shows that the STNO power level and signal purity should be improved for use in wireless communication.

To overcome challenges to communicate with the STNO, we used the OOK modulation method when directly modulating a signal of one STNO with data, as OOK modulation supports communication regardless of the signal purity. It also allows wireless communication without a PLL circuit, and with a simple structure for direct modulation. To date, there have been no reports on a realization of the frequency PLL for an STNO signal for communication. OOK can be used for communication by exploiting the advantages of the STNO for low power consumption and nano-meter scale size. Receiving of data can be easily realized simply with the function of envelope detection in the receiver. 


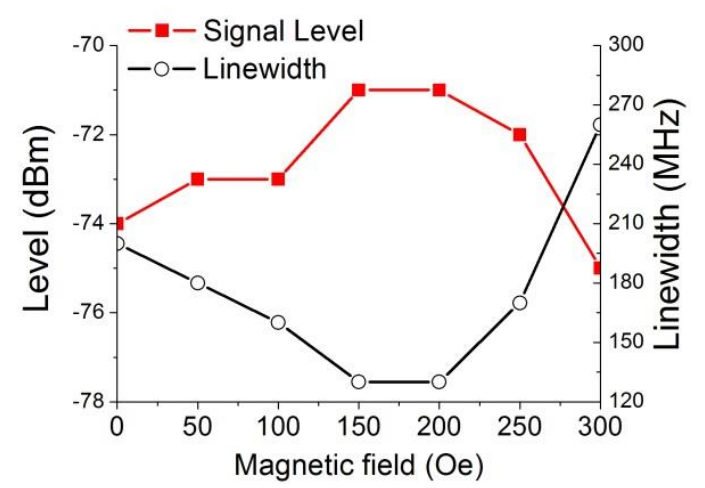

Figure 3. The signal level and linewidth of the spintronic oscillation depending on magnetic field at the condition of IDC $=1.5 \mathrm{~mA}$. ( 1 Oe (Oersted) $=1$ Gauss in air $=0.1$ millitesla).

Consequently, we target the realization of wireless communication with low power consumption under the constraint of application in near field communication due to the low level of the output signal.

Each STNO has a different frequency in the array. On this basis, a new spintronic RFdirect OOK modulation using a frequency division multiplex (FDM) technique is realized for wireless communication without interference.

The new modulation concept provides frequency modulation with an FDM by assigning each frequency at every STNO on an array structure. Amplitude is simultaneously modulated directly in the RF signal generated from the STNO with OOK on each STNO.

By simultaneously applying OOK modulation to all STNOs while generating different frequencies for multiple STNOs on an array structure, we present a new spin RF-direct OOK modulation using an FDM that modulates data together with amplitude and frequency signals for wireless communication using an STNO array.

\section{Design of the Spin Modulator}

A spintronic RF-direct OOK modulation technique using an FDM is designed by actively exploiting the strengths of the STNO, which can be easily fabricated with an array structure in a small form factor. Figure 4 shows the proposed spintronic RF-direct OOK modulation technique using an FDM concept realized with an STNO array. In particular, we designed near field communication with low power consumption by not amplifying the signal at the low power level of the STNO.

For realizing spintronic RF-direct OOK modulation using an FDM, one antenna is used in the transmitter. For easy verification, the receiver has one antenna per channel. Figure 4 shows a block diagram of wireless spintronic communication applying spintronic RF-direct OOK modulation using an FDM.

Here, Dn, $\mathrm{I}_{\mathrm{DCn}}$, and fn are nth data, current, and frequency, respectively; STNOn is the nth STNO, and MNn is a matching network for the nth frequency at the nth STNO.

We executed experiments with two STNOs to check whether wireless communication can be realized with spintronic oscillation, and also assessed whether spintronic RF-direct OOK modulation using an FDM concept was practicable. We simply modulated the signal of a spintronic oscillator with two STNOs in the array. Accordingly, the STNO, which has conditions of a magnetic field of $125 \mathrm{Oe}$ for $3.5 \mathrm{GHz}$ and 175 Oe for $4.2 \mathrm{GHz}$, generates nearly maximum output peak power of $-71 \mathrm{dBm}$ with a minimum linewidth of $130 \mathrm{MHz}$, especially has conditions of a rotation field angle of 45 degree for $3.5 \mathrm{GHz}$ and 0 degree for 4.2 $\mathrm{GHz}$ at the same magnetic field, $175 \mathrm{Oe}$; the STNO under these conditions will be used for wireless communication in this work. 


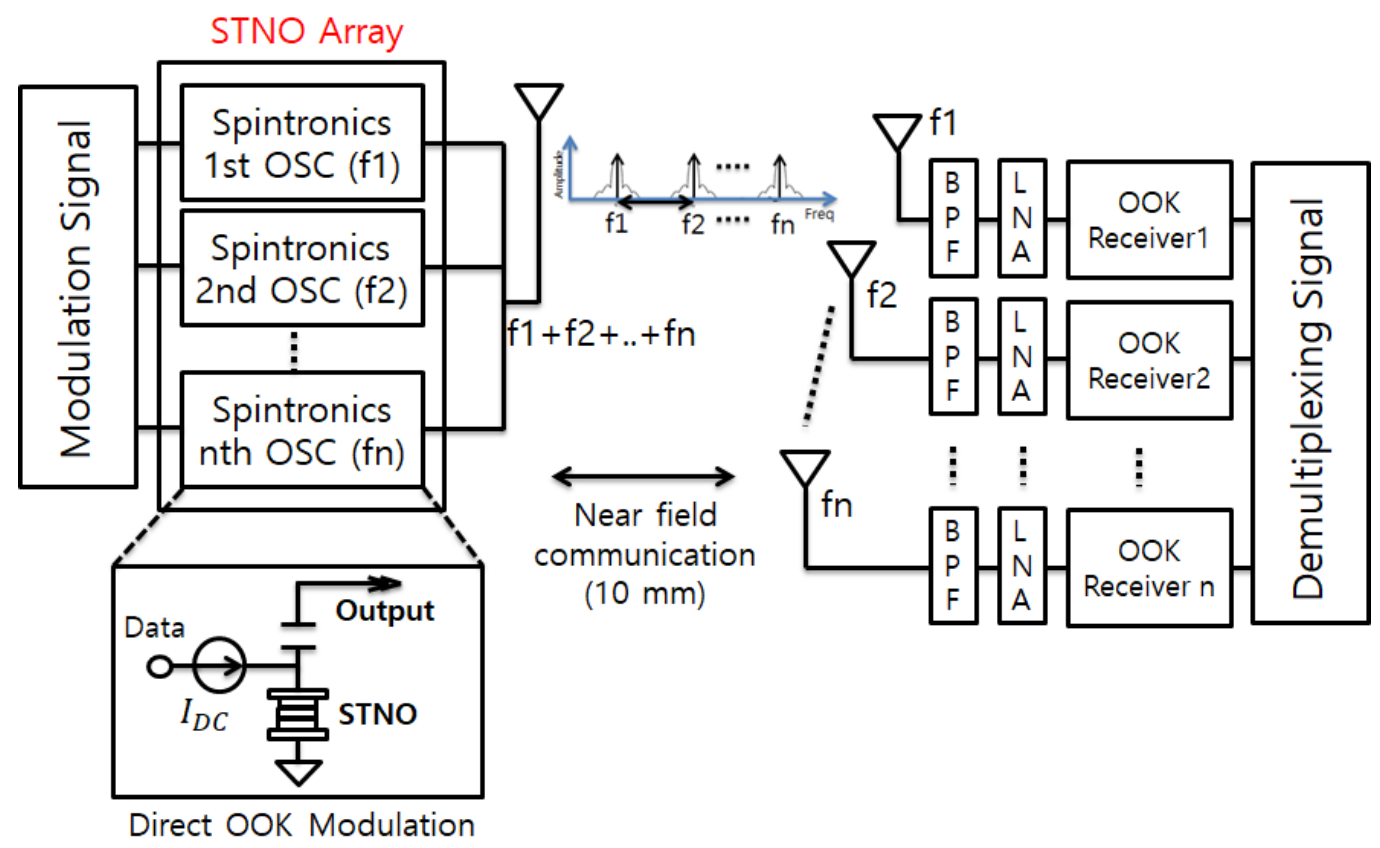

Figure 4. Block diagram of wireless spintronic communication based on an STNO array.

\subsection{Matching the Circuit on Each STNO}

The channel interval is decided between the two signals of spintronic oscillators to ensure there is no interference, considering that an STNO needs a wide separation distance between frequencies due to its poor signal purity. The signal level at the offset point corresponding with the channel separation between two channels from each carrier frequency should be selected to be below the minimum sensitivity, $-79 \mathrm{dBm}$, considering the linewidth and signal level. We thus selected frequencies of $3.5 \mathrm{GHz}$ and $4.2 \mathrm{GHz}$ with $700 \mathrm{MHz}$ channel separation for two STNOs for the channel separation to be at least three times the linewidth.

A T-junction structure is applied to the PCB for assigning the frequencies generated on two STNOs to transmit a signal in the air through a single antenna.

We designed matching networks with a low pass filter (LPF) circuit for a $3.5 \mathrm{GHz}$ STNO, and a high pass filter (HPF) circuit for a $4.2 \mathrm{GHz}$ STNO, to provide operation without interference between the STNOs. Each output port of the matching circuit was then connected with the other ports of the T-junction, taking into consideration an antenna that was situated at the center port of the T-junction. The STNO has a resistance of $80 \Omega$ at an AP state, where the STNO provides good signal oscillation [14].

An HPF circuit is applied to obtain higher isolation at $3.5 \mathrm{GHz}$ as well as low insertion loss at $4.2 \mathrm{GHz}$, and vice versa in the LPF circuit. Higher isolation and low insertion loss cause all of the signals to flow into the antenna port of the T-junction. Measured results of the T-junction are a $3 \mathrm{~dB}$ insertion loss and $7 \mathrm{dBc}$ isolation at the $3.5 \mathrm{GHz}$, and $3.5 \mathrm{~dB}$ insertion loss and $6.5 \mathrm{dBc}$ isolation at the $4.2 \mathrm{GHz}$.

Figure 5 shows the matching results for each frequency of $3.5 \mathrm{GHz}$ and $4.2 \mathrm{GHz}$. The 3.5 $\mathrm{GHz}$ matching results yield a matching result of a return loss of $-10 \mathrm{~dB}$ or less in the band range of $+/-50 \mathrm{MHz}$, while the frequency band of $4.2 \mathrm{GHz}$ or higher has a matching result of a return loss of $-5 \mathrm{~dB}$ or higher. Conversely, $4.2 \mathrm{GHz}$ matching results show that a matching result of less than a return loss of $-10 \mathrm{~dB}$ in the $+/-50 \mathrm{MHz}$ frequency band range is obtained, while a frequency band of less than $3.5 \mathrm{GHz}$ has a matching result of more than a return loss of $-5 \mathrm{~dB}$. 


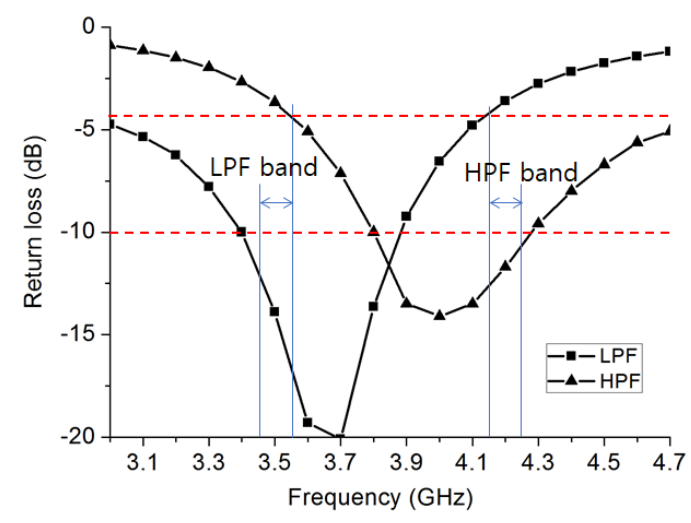

Figure 5. Matching results of the T-junction matching circuit.

The antenna is located immediately after the T-junction and it has a band from $3.1 \mathrm{GHz}$ to $5.2 \mathrm{GHz}$, and $2 \mathrm{dBi}$ gain.

The number of channels is decided with broadband oscillation and linewidth characteristics. The operation bandwidth of STNO is already sufficiently broad, and the linewidth will be improved soon. It helps a communication circuit to be designed even without any filter for isolation between channels.

\subsection{Biasing and Modulation on STNOs}

An oscillation signal is generated by the current flowing through the STNO. Current can be supplied via a current mirror circuit as a bias for a stable current supply. Figure 6 shows the schematic of biasing an STNO and modulating data through the current mirror circuit including the T-junction matching circuit on the two STNOs of the array structure.

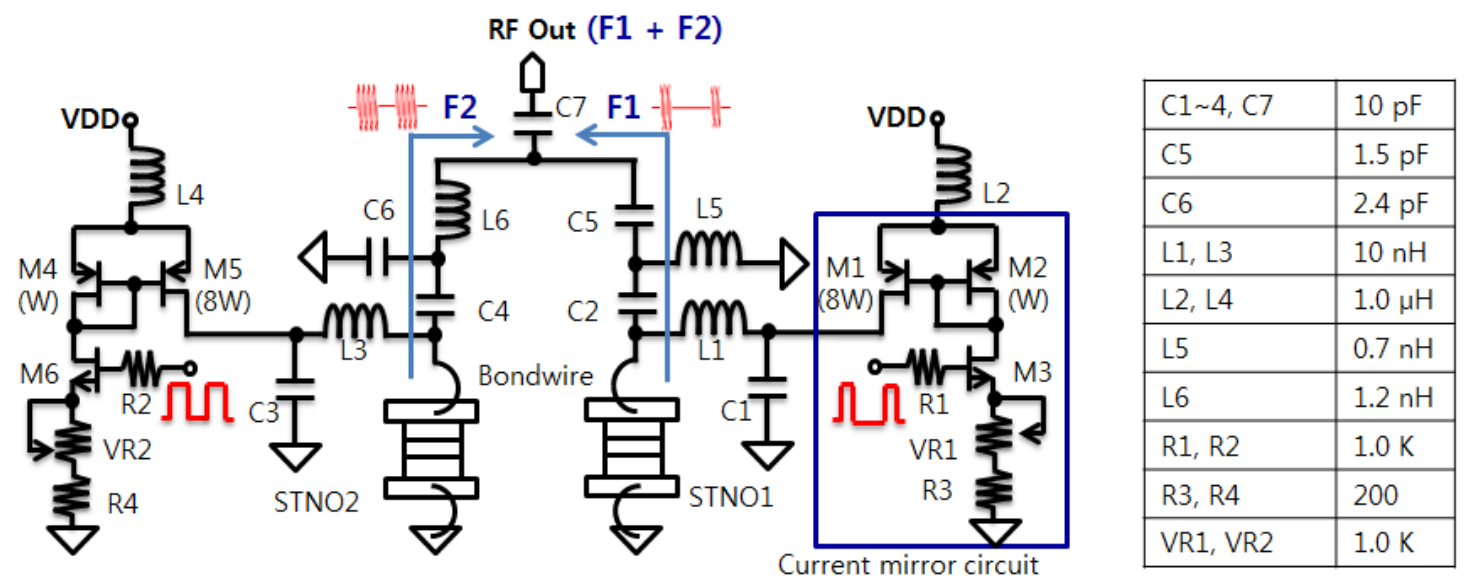

Figure 6. The schematics of the biasing an STNO and modulating a data through the current mirror circuit including the T-junction matching circuit on the two STNOs of the array structure.

We designed a current mirror circuit as a circuit supplying current with two PMOS transistors (M1, M2, M4, and M5), as shown in Figure 6. The ratio of the transistor width for the current mirror circuit must be set at 1:8 to reduce the leakage current to 1/8th of the current flowing through the STNO.

The STNO is easily broken by the breakdown current, as the magnetic tunnel junction (MTJ) is operated with a tunneling effect in the inserted insulator as a non-magnetic layer. A variable resistor (VR1 or VR2) is added in the current mirror circuit to limit the current for safe operation together with R3 and R4. The value of VR1 or VR2 is set for the current flowing through STNO to flow as around $1.5 \mathrm{~mA}$ in this work.

The supplied current drives the STNO to generate oscillation. We then modulate the STNO with the OOK method, where the current supplied from the current mirror circuit is 
switched on/off. This function is executed through a NMOS transistor (M3 or M6) switch that enables operation of the current mirror circuit.

\subsection{Simulation on an OOK Modulation Rate in the STNO}

Although $[11,15,16]$ reported that current clearly modulates pulse repetition frequency (PRF) up to $5 \mathrm{MHz}$, and an STNO has a fast settling time, nearly $1 \mathrm{~ns}$, the usable frequency of PRF is limited by the bias delay driving current at the transmitter, and by a falling time of $180 \mathrm{~ns}$ and a rising time of $100 \mathrm{~ns}$ for detection at the receiver in the experiments reported in this work.

By introducing the Landau-Lifshitz-Gilbert-Slonczewski (LLGS) equation, we conducted a simulation to assess how the rising and falling time of oscillation for the MTJ pillar is delayed by the effect of the bias circuit to drive the STNO in the transmitter. This condition is that each matching circuit is perfectly matched to the antenna and isolated each other between two ports of an STNO. Magnetic dynamics of the free layers of an STNO are found from the LLGS equation $[12,13,17,18]$. The LLGS equation used here is given as Equation (1).

$$
\frac{d \hat{m}}{d t}=-\gamma \hat{m} \times H_{e f f}+\alpha \hat{m} \times \frac{d \hat{m}}{d t}+\gamma a_{J} \hat{m} \times(\hat{m} \times \hat{\sigma})+\gamma b_{J} \hat{m} \times \hat{\sigma}
$$

where $\hat{m}$ is the unit vector of free layer magnetization, $\gamma$ is the gyromagnetic ratio, $\alpha$ is the Gilbert damping parameter, and $\hat{\sigma}$ is the unit vector of pinned layer magnetization. The effective magnetic field, $H_{e f f}$, is composed of an applied magnetic field, $H_{a p p}$, a uniaxial anisotropy field, $H_{k}$, and the demagnetization filed, $H_{d}$. Therefore, the effective magnetic field can be written as:

$$
H_{\text {eff }}=H_{\text {app }} \hat{x}+H_{k}(\hat{m} \cdot \hat{x}) \hat{x}-H_{d}(\hat{m} \cdot \hat{z}) \hat{z}
$$

where $\hat{x}$ and $\hat{z}$ are the unit vectors along the $\mathrm{x}$ and $\mathrm{z}$ axes, respectively.

The first part of Equation (1) is the Larmor precession, the second is Gilbert damping, the third is in-plane spin torque which is competes with the Gilbert damping, and the last is the perpendicular spin torque equation $[12,17,19] . a_{J}$ and $b_{J}$ in the third and the fourth part of Equation (1), respectively, are the current density, which is related to the scale of spin torque. $a_{J}$ is the in-plane torque efficiency and $b_{J}$ is the perpendicular torque efficiency. The in-plane field torque, $a_{J}$, competes with the damping $(\alpha)$ whereas the perpendicular torque, $b_{J}$, acts similar to a magnetic field [20].

We assumed that the capacitance is located parallel with the STNO due to the bias circuit, and there are ideally no parasitic components except the internal resistance of the STNO having a value between $48 \Omega$ and $80 \Omega$. Figure 7 shows the simplified circuit driving the STNO for the simulation.

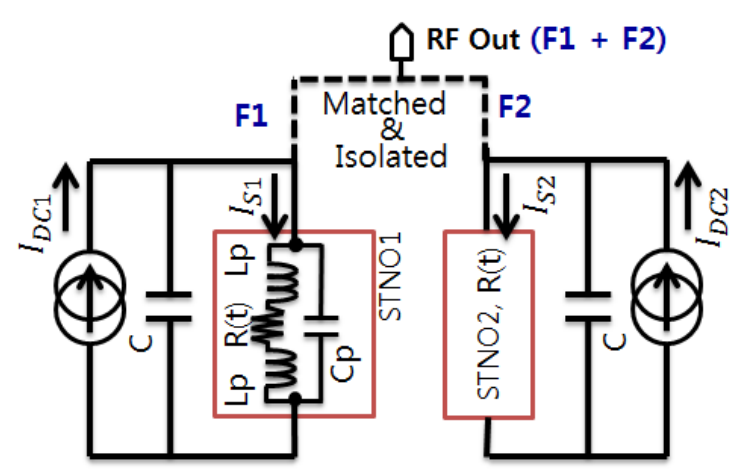

Figure 7. Simplified circuit driving the STNO. (C is the capacitance located in parallel with the STNO, and $L p, C p$, and $R(t)$ are the parasitic inductor, parasitic capacitor, and internal resistance of the STNO, respectively). 
In the MTJ structure of $100 \times 90 \mathrm{~nm}^{2}$ with a $1 \mathrm{~nm}$ tunneling gap, the parasitic capacitance is around 0.1 femto-Farad, and the parasitic inductor is around 1 pico-Henry in the condition of $1.5 \mathrm{~mA}$ operation. These parasitic components can be neglected in the equivalent circuit and the numerical equations of a $3 \sim 5 \mathrm{GHz}$ band circuit.

We also assumed that the STNO directly modulates the on/off signal of the DC current for operation of OOK modulation. The high or low DC current signal, $I_{D C}$, has a level of $1.5 \mathrm{~mA}$ or ground for a logic level; however, we made this signal have a $1 \mathrm{~ns}$ delay time for the input of the bias circuit to drive the STNO. This reason is for obtaining settling time from the response of the on/off current signal into the real delay value, as the function generator, AFG3251C, outputs a rectangular wave with a 1 ns delay time into the on/off DC signal [21].

The current induced into the STNO, $I_{S}$, can be expressed as Equation (3).

$$
d\left[I_{S}(t)\right] / d t=\left(I_{D C}-I_{S}(t)-C I_{S}(t) d[R(t)] / d t\right) /(C R(t))
$$

where $C$ is the capacitance located in parallel with the STNO, and $R(t)$ is the internal resistance of the STNO.

$I_{S}$ is modulated into the STNO after being delayed by the internal resistance of the STNO and the capacitance, which is a main part of the bias-T circuit, as delineated in Equation (3). In this equation, we defined the internal resistance of the STNO, as expressed in Equation (4). The parallel MR, $R_{P}$, of the fabricated MTJ pillar is $48 \Omega$, and the antiparallel MR, $R_{A P}$, is $80 \Omega$ according to the magnetization angular momentum of the free layer for MTJ pillar.

$$
R(t)=\left(R_{A P}+R_{P}\right) / 2-\left(R_{A P}-R_{P}\right) / 2 \cos [\theta(t)]
$$

The transient delay for an STNO oscillation signal obtained from a modulated signal is solved from the LLGS equation, given as Equation (1). $b_{J} / a_{J}$ is assigned as 0.1 in our simulation. The simulation of Equation (1) is performed by the Runge-Kutta algorithm. The parameters used in our simulation are enumerated as follows: $\alpha=0.01$, $\gamma=1.85 \times 10^{11} \mathrm{~Hz} / \mathrm{T}[20]$.

If the RF signal flows through the bias circuit path and the modulating signal output circuit path, the RF signal loss decreases as the capacitor value between the bias circuit and the STNO decreases.

Additionally, this capacitor value is related to the setting time of the STNO oscillation signal.

In this paper, to reduce RF signal loss while minimizing latency, the capacitor between the bias circuits of STNO is selected as 10pF. The simulation results for the 10pF circuit conditions resulted in RF signal loss of $-32 \mathrm{~dB}$ and setting time of $16 \mathrm{~ns}$. As RF signal loss is weak, the capacitor value is set to $10 \mathrm{pF}$ based on the setting time. The resulting communication transfer rate was calculated to be constrained to $3.38 \mathrm{Mbps}(=1 / 296 \mathrm{~ns}$, $16 \mathrm{~ns}$ for transmitter, $100 \mathrm{~ns}+180 \mathrm{~ns}=280 \mathrm{~ns}$ for receiver).

Figure 8 is the time domain signal transmitted from the transmitter after transmitting OOK signals of the STNO, and signals received from the receiver located at a distance of $10 \mathrm{~mm}$ from the transmitter after demodulating the signal.

As the third part of equation, the in-plane toque component, in the LLGS equation is affected by the long initial start time, long lag time occurs before generating a spin toque signal. Therefore, we used the measurement AFG3251C for short delayed modulation signal under $1 \mathrm{~ns}$, resulting in the short rising time into $10 \mathrm{~ns}$. Figure 8 displays the measurement results observed from the RF monitor after modulating $1 \mathrm{MHz}$ data together with the simulation results. The experiment results show that a data rate of up to $2 \mathrm{Mbps}$ is obtained at each frequency. This shows that a data rate of up to $4 \mathrm{Mbps}$ is acquired by spintronics RF-direct OOK modulation using an FDM with two STNOs in the array. It should be noted that we proposed a new method, making it possible to easily increase the data rate by actively applying an STNO array structure. 


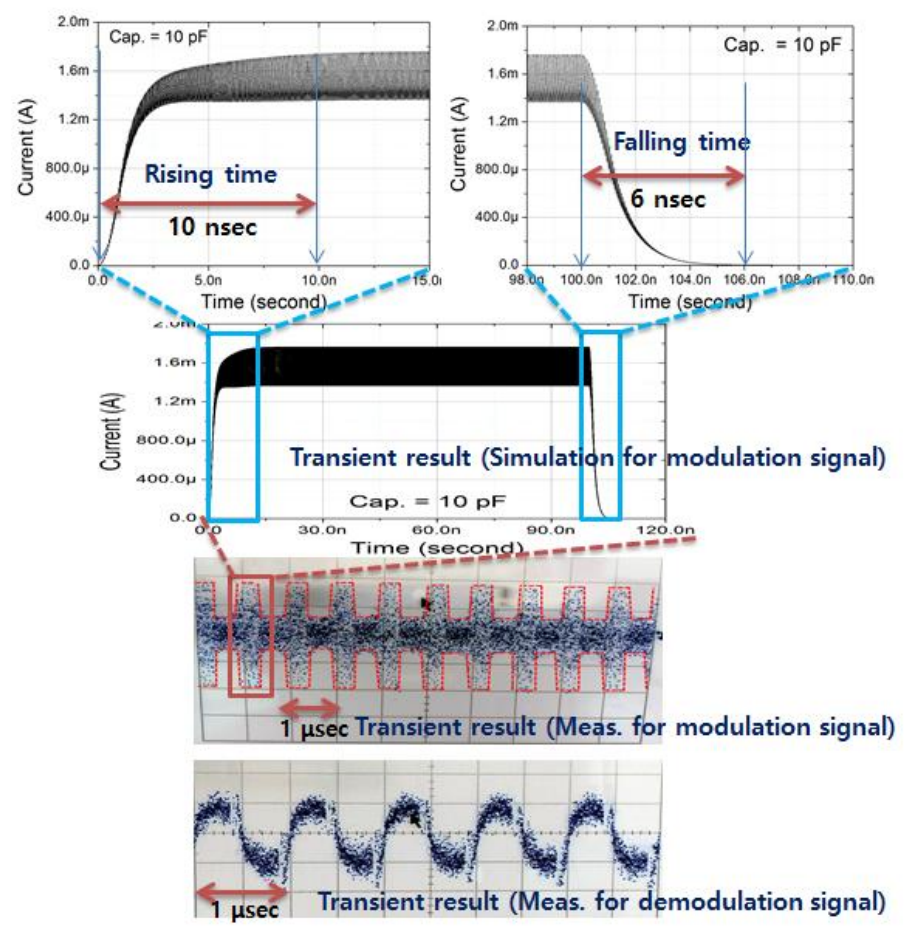

Figure 8. Transient results comparing simulation and measurement results for the modulation.

\section{Design of the Spin Demodulator}

As shown in Figure 4, we applied a broadband antenna to the receiver to accommodate all channels with the same type of antenna, and located a band pass filter (BPF) immediately after each antenna in order to separately select and detect the target signal on a channel assigned at each STNO. The antenna used is ANT1085-4R1-01A from TDK, and the bandwidth of the antenna used is sufficiently covered from $3.1 \mathrm{GHz}$ to $5.2 \mathrm{GHz}$, with an antenna gain of $2 \mathrm{dBi}$. BPF applied Johansson's 3600BP for the $3.5 \mathrm{GHz}$ band and Taiyo Yuden's 4224-to- 4752 for the $4.2 \mathrm{GHz}$ band. BPF has an insertion loss of $1.5 \mathrm{~dB}$ and $2.0 \mathrm{~dB}$ for $3.5 \mathrm{GHz}$ and $4.2 \mathrm{GHz}$, respectively. In particular, isolation between channels is important for receiving a signal without interference in the condition of two channels on a single board. Isolation for $\mathrm{BPF}$ is above $35.5 \mathrm{~dB}$ at $4.2 \mathrm{GHz}$ when transmitting a $3.5 \mathrm{GHz}$ signal, and above $14.8 \mathrm{~dB}$ at $3.5 \mathrm{GHz}$ when transmitting a $4.2 \mathrm{GHz}$ signal.

For good sensitivity of the receiver, a Low Noise Amplifier (LNA) was placed immediately after each BPF. The LNA used was applied to Avago's MGA86563. The LNA has a noise figure of $1.8 \mathrm{~dB}$ and $2.0 \mathrm{~dB}$ at $3.5 \mathrm{GHz}$ and $4.2 \mathrm{GHz}$, respectively, and the gain is measured as $20.0 \mathrm{~dB}$ and $17.1 \mathrm{~dB}$, respectively. In particular, isolation between channels is important for receiving a signal well without interference in a two channels condition on a single board. Figure 9 shows the isolation and gain characteristics obtained from measurement, together with the BPF and LNA. The measured results are a gain of $20.0 \mathrm{~dB}$ and $17.1 \mathrm{~dB}$, and isolation of $14.8 \mathrm{~dB}$ and $35.5 \mathrm{~dB}$ at $3.5 \mathrm{GHz}$ and $4.2 \mathrm{GHz}$, respectively, considering BPF loss of $1.5 \mathrm{~dB}$ and $2.0 \mathrm{~dB}$.

We additionally could situate the attenuator of $-8 \mathrm{~dB}$ after the LNA at the $3.5 \mathrm{GHz}$ path to secure more isolation even in the wired condition between the transmitter and receiver.

In this study, we targeted wireless communication under $10 \mathrm{~mm}$ distance between the transmitter and receiver, considering the used MTJ pillar has approximately a $-71 \mathrm{dBm}$ output power level.

The link-budget for short distance range communication is calculated with the bandwidth, taking into consideration the signal purity as well as the power level, antenna characteristics, path loss, and noise figure in the receiver. Table 1 shows the link-budget of the wireless communication for $10 \mathrm{~mm}$ distance when making the STNO operate at 
3.5 GHz and 4.2 GHz. The link budget is calculated with the air loss as $5 \mathrm{~dB}$ in a distance of $10 \mathrm{~mm}$.

The power level transmitted through the antenna is $-72.0 \mathrm{dBm}$ and $-72.5 \mathrm{dBm}$ at 3.5 GHz and 4.5 GHz, and the thermal noise level is $-92.9 \mathrm{dBm}$ in the $130 \mathrm{MHz}$ STNO linewidth. The SNR in the transmitter is thus $20.9 \mathrm{~dB}(=-72.0+92.9)$ and $20.4 \mathrm{~dB}$ $(=-72.5+92.9)$, respectively. The transmission margin is $11.9 \mathrm{~dB}(=20.9-9.0)$ and $11.4 \mathrm{~dB}(=20.4-9.0)$, as the required SNR is $9.0 \mathrm{~dB}$ for the $10^{-3}$ bit error rate (BER) in the OOK system.

In the receiver we tested at a distance $10 \mathrm{~mm}$, the system noise figure is $4.3 \mathrm{~dB}$ and $4.5 \mathrm{~dB}$ at $3.5 \mathrm{GHz}$ and $4.2 \mathrm{GHz}$, respectively, and the minimum sensitivity of the receiver is optimally $-79.6 \mathrm{dBm}$ and $-79.4 \mathrm{dBm}$ at $3.5 \mathrm{GHz}$ and $4.2 \mathrm{GHz}$, respectively, considering the system NF and the required SNR. The transmission margin is then $4.6 \mathrm{~dB}$ $(=-75.0+79.6)$ and $3.9 \mathrm{~dB}(=-75.5+79.4)$ at $3.5 \mathrm{GHz}$ and $4.2 \mathrm{GHz}$, respectively. The margin for $3.9 \mathrm{~dB}$ corresponds to supporting transmission up to $15.8 \mathrm{~mm}$ distance in a $4.2 \mathrm{GHz}$ communication system.

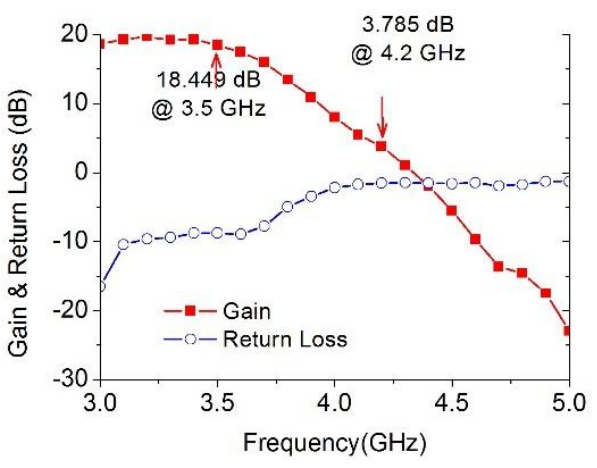

(a)

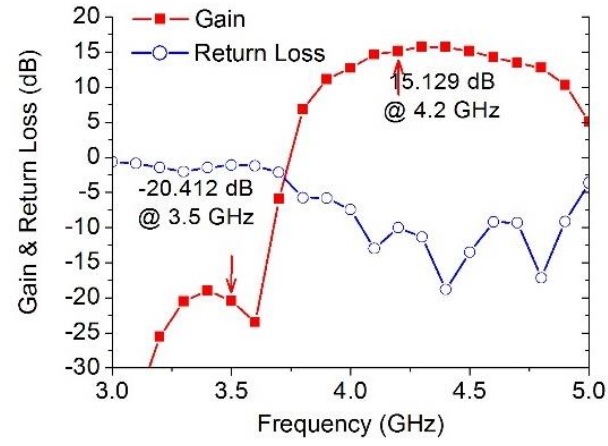

(b)

Figure 9. Measurement results of the BPF and LNA for the $3.5 \mathrm{GHz}$ and $4.2 \mathrm{GHz}$ signal paths. (a) Results of the gain and isolation at $3.5 \mathrm{GHz}$ when transmitting a $4.2 \mathrm{GHz}$ signal (20.0 dB for gain, $14.8 \mathrm{~dB}$ for isolation) (b) Results of the gain and isolation at $4.2 \mathrm{GHz}$ when transmitting a $3.5 \mathrm{GHz}$ signal (17.1 dB for gain, $35.5 \mathrm{~dB}$ for isolation). 
Table 1. The specification and the signal flow in the receiver and transmitter.

\begin{tabular}{|c|c|c|c|c|c|c|c|c|}
\hline \multirow{2}{*}{$\begin{array}{l}\text { Parameters } \\
\text { Signal Flow }\end{array}$} & \multicolumn{3}{|c|}{ Transmitter } & \multirow[b]{2}{*}{ Air } & \multicolumn{4}{|c|}{ Receiver } \\
\hline & STNO Output & T-Junction & Antenna & & Antenna & BPF & LNA & $\begin{array}{l}\text { Demod } \\
\text { Sensitivity }\end{array}$ \\
\hline $\begin{array}{c}\text { Specification } \\
(3.5 \mathrm{GHz} / 4.2 \mathrm{GHz})\end{array}$ & $-71 \mathrm{dBm}$ & $\begin{array}{c}-3.0(\mathrm{LPF}) /-3.5 \\
\mathrm{~dB}(\mathrm{HPF})\end{array}$ & $\begin{array}{c}2 \mathrm{dBi} \\
\text { (covering } 3.1 \text { to } 5.2 \\
\mathrm{GHz} \text { ) }\end{array}$ & $\begin{array}{c}20 \log \frac{4 \pi \mathrm{d}}{\lambda}=-5 \mathrm{~dB} \\
@ 10 \mathrm{~mm}\end{array}$ & $\begin{array}{c}2 \mathrm{dBi} \\
\text { (covering } 3.1 \text { to } 5.2 \\
\mathrm{GHz} \text { ) }\end{array}$ & $\begin{array}{c}-1.5 / \\
-2.0 \mathrm{~dB}\end{array}$ & $\begin{array}{c}20 / 17.1 \mathrm{~dB} \\
(12 * / 17.1 \mathrm{~dB})\end{array}$ & \multirow{3}{*}{$\begin{array}{l}\text { Min. sensitivity: } \\
-79.6 \mathrm{dBm} @ 3.5 \\
\mathrm{GHz} /-79.4 \mathrm{dBm} \\
@ 4.2 \mathrm{GHz}\end{array}$} \\
\hline $\begin{array}{l}\text { Signal flow 3.5/4.5 } \\
\text { GHz Level (dBm) }\end{array}$ & -71 & $\begin{array}{l}-74 / \\
-74.5\end{array}$ & $\begin{array}{l}-72 / \\
-72.5\end{array}$ & $\begin{array}{l}-77 / \\
-77.5\end{array}$ & $\begin{array}{l}-75 / \\
-75.5 \\
\end{array}$ & $\begin{array}{c}-76.5 / \\
-77.5\end{array}$ & $\begin{array}{l}-64.5 / \\
-60.4 \\
\end{array}$ & \\
\hline $\begin{array}{c}\text { System Gain (3.5 } \\
\text { GHz/4.2 GHz) }\end{array}$ & \multicolumn{3}{|c|}{$-1 \mathrm{~dB} /-1.5 \mathrm{~dB}$} & \multicolumn{4}{|c|}{$14.5 \mathrm{~dB} / 17.1 \mathrm{~dB}$} & \\
\hline $\begin{array}{l}\text { Isolation at } 3.5 \mathrm{GHz} \\
\text { from } 4.2 \mathrm{GHz}\end{array}$ & \multirow{2}{*}{$-71 \mathrm{dBm}$} & $\begin{array}{c}-10 \mathrm{~dB} \\
(-81 \mathrm{dBm})\end{array}$ & $\begin{array}{c}2 \mathrm{dBi} \\
(-79 \mathrm{dBm})\end{array}$ & $\begin{array}{l}0 \mathrm{~dB} \text { (on wired } \\
\text { condition) }\end{array}$ & $\begin{array}{c}2 \mathrm{dBi} \\
(-77 \mathrm{dBm})\end{array}$ & $\begin{array}{c}-14.8 \mathrm{~dB} \\
(-91.8 \mathrm{dBm})\end{array}$ & $12 \mathrm{~dB}$ & $-79.8 \mathrm{dBm}$ \\
\hline $\begin{array}{l}\text { Isolation at } 4.2 \mathrm{GHz} \\
\text { from } 3.5 \mathrm{GHz}\end{array}$ & & $\begin{array}{c}-10 \mathrm{~dB} \\
(-81 \mathrm{dBm})\end{array}$ & $\begin{array}{c}2 \mathrm{dBi} \\
(-79 \mathrm{dBm})\end{array}$ & $\begin{array}{l}0 \mathrm{~dB} \text { (on wired } \\
\text { condition) }\end{array}$ & $\begin{array}{c}2 \mathrm{dBi} \\
(-77 \mathrm{dBm})\end{array}$ & $\begin{array}{l}-35.5 \mathrm{~dB}(-112.5 \\
\mathrm{dBm})\end{array}$ & $17.1 \mathrm{~dB}$ & $-95.4 \mathrm{dBm}$ \\
\hline Noise level (kTB) & \multicolumn{8}{|c|}{$\begin{array}{c}\text { kTB: }-92.9 \mathrm{dBm} @ 130 \mathrm{MHz} \text { Bandwidth } \\
\text { (k: Boltzmann's constant, T: Temperature, B: Bandwidth) }\end{array}$} \\
\hline
\end{tabular}

* The $-8 \mathrm{~dB}$ attenuator is added right after the LNA only at a $3.5 \mathrm{GHz}$ path to secure isolation. 


\section{Measurement Results}

Figure 10 shows the block diagram for the test and the measurement setup environment. To perform the test for wireless communication, the fabricated spintronics array transmitter is placed on a C-type magnetic closed circuit to apply a magnetic field around the DUT. Constant current is applied to the electromagnet by a precision current source, and a fringing field is generated depending on the current quantity. The direction of the magnetic field, rotation field angle, is also changed by manually making the C-type magnetic closed circuit rotate, as shown in Figure 10. Current quantities were supplied by changing the current corresponding to each STNO bonded to the $50 \mathrm{ohm}$ line through the bias-tee, allowing the required magnetic field amplitude and phase to be obtained by limiting the current to protect the STNO.

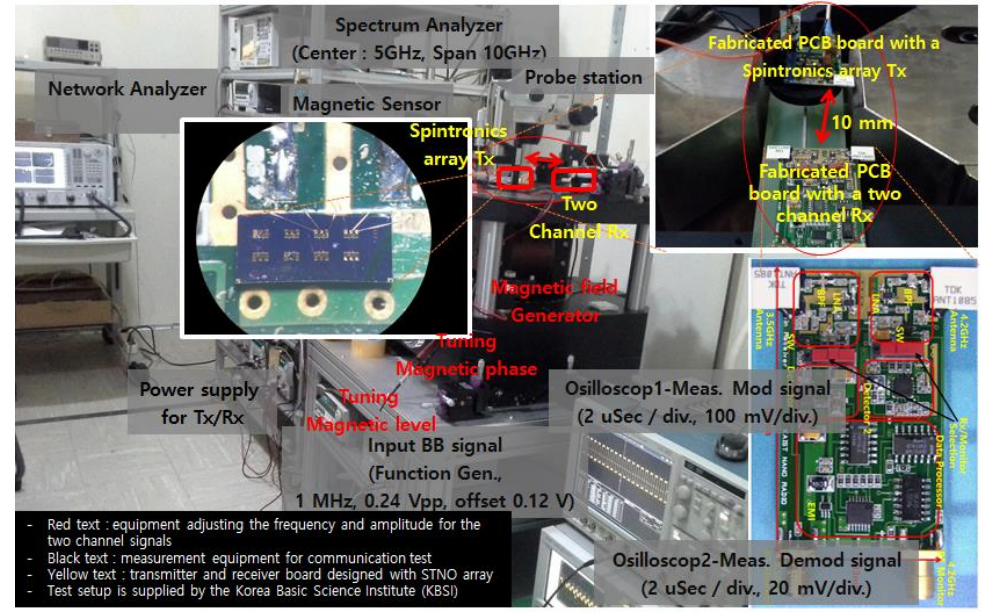

(a)

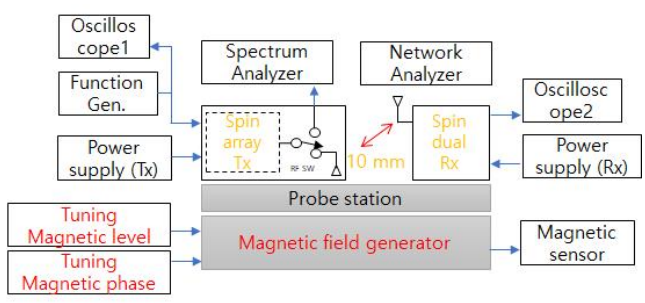

(b)

Figure 10. Measurement settings for two-channel receivers and spintronic RF-Direct On-Off keying modulation. (a) Block-diagram of the test setup, and (b) test environment picture.

Figure 10 shows the spintronics array Tx bonded with STNOs structured in a $2 \times 4$ array for transmitting multi-channel signals on the magnetic field generator. We selected two out of the eight STNOs and set the target frequency by controlling the magnitude of the magnetic field, the rotation field angle of the applied magnetic field to the STNO, and the current quantity based on STNO operation [22].

Figure 10 also shows a photo of the two channels receiver, each of which has an antenna, BPF, LNA, and detector, and the receivers are designed into the assigned frequency band excluding the antennas, which cover both channels. We situated an RF switch between the LNA and the detector to monitor the received signal at the assigned RF monitor port. The RF switch is controlled by a dip switch.

Under these design boards, we carried out the test of wireless communication by the spintronic RF-direct OOK modulation using an FDM with two STNOs in the array.

The receiver is located at a distance of $10 \mathrm{~mm}$ from the transmitter. Under these measurement conditions, we tested the operation of the transmission on two frequencies modulating OOK into one antenna.

In Figure 10, red text indicates the equipment to adjust the frequency and amplitude for the two channel signals, black text is the measurement equipment for the communication test, and yellow text is the transmitter and receiver board designed with the STNO array. The test setup was supplied by the Korea Basic Science Institute (KBSI, Deajeon, Korea).

The measurement results show that a data rate of up to $4 \mathrm{Mbps}$ is obtained at array STNO, and the power consumption is under $3 \mathrm{~mW}$ per STNO at these measurement conditions. It is observed that $0.18 \mathrm{~mW}$ power consumption occurs at one STNO. The value of $0.9 \mathrm{~nJ} /$ bit obtained from the power consumption result of spintronic modulated radio 
technology is not a remarkable achievement, as it uses commercial circuits supplied with 2 VDC. Nevertheless, this is the first successful trial of communication with spintronic technology in the array structure.

Based on this transmission and reception result, the UART interface was used as a modulation input to obtain and transmit the serial signal wirelessly, and it was confirmed that the image was displayed successfully. In this experiment, the maximum transmission rate of UART was 115,200 bps.

These circuits will be fabricated in the near future, together with a sub-10 nm scaled STNO array CMOS technology such as a magnetoresistive random-access memory (MRAM). The size and power consumption of the wireless technology of spintronic modulation consequently approach those of the core block, the STNO.

STNOs have low power consumption behavior characteristics, which are more than 10 times less than other published results [23-25] designed for lower milliwatt operation, as shown in Table 2. Notably, the wireless technology of spintronic modulation could be designed with much smaller size, as shown in Table 2, than even a ring VCO which is designed without an inductor [25], as it is operated independently as a nano-scaled resonator.

Table 2. The STNO Performance comparison with other Works.

\begin{tabular}{ccccc}
\hline Ref. & LC VCO [23] & LC VCO [24] & Ring VCO [25] & STNO (This Work) \\
\hline $\begin{array}{c}\text { Oscillation frequency } \\
\text { (GHz) }\end{array}$ & $4.0 \sim 4.34$ & $13 \sim 19$ & $0.11 \sim 0.55$ & $2.1 \sim 4.9$ \\
\hline Tune range (\%) & $8.2 \%$ & $37.5 \%$ & $133 \%$ & $80 \%$ \\
\hline Phase noise & $-113.6 \mathrm{dBc} / \mathrm{Hz} @ 1$ & $-113 \mathrm{dBc} / \mathrm{Hz} @ 1 \mathrm{MHz}$ & $-71 \mathrm{dBc} / \mathrm{Hz}$ & $-63 \mathrm{dBc} / \mathrm{Hz} @ 130$ \\
$\mathrm{MHz}$ & 3.69 & 0.18 \\
\hline Core Pdc $(\mathrm{mW})$ & 1.8 & 24 & $0.01 \mathrm{~mm}^{2}$ & $0.009 \mu \mathrm{m}^{2}$ \\
\hline Size & $0.19 \mathrm{~mm}^{2}$ & $0.14 \mathrm{~mm}^{2}$ & \\
\hline
\end{tabular}

The STNO and a ring VCO has a wider tuning range than LC VCO, whose frequency range is limited by the resonance of the inductor and capacitor. A ring VCO has broadband operating characteristics, but generally has low frequency oscillation characteristics, and it is difficult to generate RF frequencies. However, STNOs can obtain frequencies up to RF and millimeter waves. This allows design as a communication structure with direct conversion.

The characteristics of a low power consumption, wide tuning range, and small size provide flexibility of design for array structure. In spite of these characteristics of STNO, the spectral purity of the STNO is much worse than others. We thus proposed a wireless communication with STNO-adopted OOK modulation which can overcome the problems of poor spectral purity.

\section{Conclusions}

We demonstrated a new communication technique with spintronics technology. It is the first report to implement wireless communication by applying STNO array to modulate frequencies and amplitudes simultaneously. The obtained results demonstrate the possibility of increasing data rates remarkably by using an STNO array, which features a wide band range even exceeding 100\%, low DC consumption at the micro-watt level, and nano-sized realization. However, many barriers must still be surmounted. In particular, the linewidth of STNO's oscillation signal was $130 \mathrm{MHz}$, so in this paper, only two STNOs could be transmitted using the on-off Keying modulation to secure twice the transmission speed. As research is currently under way to improve linewidth to $\sim \mathrm{MHz}$, it is believed that RF-Direct on-off Keying Modulation can be performed on a number of STNOs. It would be easy to increase the transmission speed. Increased transmission speed in the big data environment is an essential requirement for communication. This paper presents a technology that can easily speed up transmission data in the array structure of STNO. 
Furthermore, as STNOs are capable of implementing nano-size in low power consumption in $\mu \mathrm{W}$ and array structures, the proposed plan is expected to run competitively on highspeed, low-power, and small-scale near field communication (NFC) at relatively low cost. In addition, STNO's output level is expected to increase even at low power consumption in the future, so it can be applied not only to proximity applications but also to various communication applications.

Author Contributions: Conceptualization, I.-Y.O.; validation, I.-Y.O.; formal analysis, M.-S.K.; Writing-review and editing, M.-S.K. and K.-S.K.; Funding acquisition and supervision, C.-H.C. All authors have read and agreed to the published version of the manuscript.

Funding: This work was supported by the Sun Moon University Research Grant of 2021.

Acknowledgments: The authors are also pleased to acknowledge a valuable co-operation work with KIST and KBSI.

Conflicts of Interest: The authors declare no conflict of interest.

\section{References}

1. Arun, R.; Gopal, R.; Chandrasekar, V.K.; Lakshmanan, M. Frequency enhancement and power tunability in tilted polarizer spin-torque nano-oscillator. J. Appl. Phys. 2020, 127, 153903. [CrossRef]

2. Zeng, Z.; Finocchio, G.; Zhang, B.; Amiri, P.K.; Katine, J.A.; Krivorotov, I.N.; Huai, Y.; Langer, J.; Azzerboni, B.; Wang, K.L.; et al. Ultra low-current-density and bias-field-free spin-transfer nano-osicllator. Nat. Sci. Rep. 2013, 3, 1426. [CrossRef] [PubMed]

3. Ruiz-Calaforra, A.; Purbawati, A.; Brächer, T.; Hem, J.; Murapaka, C.; Jiménez, E.; Mauri, D.; Zeltser, A.; Katine, J.A.; Cyrille, M.-C.; et al. Frequency shift keying by current modulation in a MTJ-based STNO with high data rate. Appl. Phys. Lett. 2017, 111, 082401. [CrossRef]

4. Sharma, R.; Sisodia, N.; Iacocca, E.; Awad, A.A.; Åkerman, J.; Muduli, P.K. A high-speed single sideband generator using a magnetic tunnel junction spin torque nano-oscillator. Sci. Rep. 2017, 7, 13422. [CrossRef]

5. Litvinenko, A.; Sethi, P.; Murapaka, C.; Jenkins, A.; Cros, V.; Bortolotti, P.; Ferreria, R.; Dieny, B.; Ebels, U. Analog and digital phase modulation of spin torque nano-oscillators. arXiv 2019, arXiv:1905.02443.

6. Pufall, M.R.; Rippard, W.H.; Kaka, S.; Silva, T.; Russek, S.E. Frequency modulation of spin-transfer oscillators. Appl. Phys. Lett. 2005, 86, 082506. [CrossRef]

7. Consolo, G.; Puliafito, V.; Finocchio, G.; Lopez-Diaz, L.; Zivieri, R.; Giovannini, L.; Nizzoli, F.; Valenti, G.; Azzerboni, B. Combined Freqeuncy-Amplitude Nonlinear Modulation Theory and Application. IEEE Trans. Magn. 2010, 46, 3629-3634. [CrossRef]

8. Quercia, A.; D'Aquino, M.; Scalera, V.; Perna, S.; Serpico, C. Normal form of nonlinear oscillator model relevant to spin-torque nano-oscillator theory. Phys. B Condens. Matter 2018, 549, 87-90. [CrossRef]

9. Oh, I.-Y.; Park, S.-Y.; Kang, D.-H.; Park, C.S. Wireless Spintronics Modulation with a Spin Torque Nano-Oscillator (STNO) Array. IEEE Microw. Wirel. Compon. Lett. 2014, 24, 502-504. [CrossRef]

10. Choi, H.S.; Kang, S.Y.; Cho, S.J.; Oh, I.-Y.; Shin, M.; Park, H.; Jang, C.; Min, B.-C.; Kim, S.-I.; Park, S.-Y.; et al. Spin nano-oscillatorbased wireless communication. Nat. Sci. Rep. 2014, 4, 5486. [CrossRef]

11. Krivorotov, I.N.; Emley, N.C.; Sankey, J.C.; Kiselev, S.I.; Ralph, D.C.; Buhrman, R.A. Time-Domain measurements Of Nanomagnet Dynamics Driven by Spin-Transfer Torques. Science 2005, 307, 228-231. [CrossRef] [PubMed]

12. Slonczewski, J. Current-driven excitation of magnetic multilayers. J. Magn. Magn. Mater. 1996, 159, L1-L7. [CrossRef]

13. Deac, A.M.; Fukushima, A.; Kubota, H.; Maehara, H.; Suzuki, Y.; Yuasa, S.; Nagamine, Y.; Tsunekawa, K.; Djayaprawira, D.D.; Watanabe, N. Bias-driven high-power microwave emission from MgO-based tunnel magnetoresistance devices. Nat. Phys. 2008, 4, 803-809. [CrossRef]

14. Oh, I.-Y.; Park, S.-Y.; Park, C.-S. A Transistor Array Bank (TR BANK) for a Digitally Controlled Spin Torque Oscillator. Microw. Opt. Technol. Lett. 2014, 56, 1991-1994. [CrossRef]

15. Manfrini, M.; Devolder, T.; Kim, J.-V.; Crozat, P.; Chappert, C.; Van Roy, W.; Lagae, L. Frequency shift keying in vortex-based spin torque oscillators. J. Appl. Phys. 2011, 109, 083940. [CrossRef]

16. Keller, M.W.; Kos, A.B.; Silva, T.; Rippard, W.H.; Pufall, M.R. Time domain measurement of phase noise in a spin torque oscillator. Appl. Phys. Lett. 2009, 94, 193105. [CrossRef]

17. Berger, L. Emission of spin waves by a magnetic multilayer traversed by a current. Phys. Rev. B 1996, 54, 9353-9358. [CrossRef]

18. Zhou, Y.; Akerman, J. Perpendicular spin torque promotes synchronization of magnetic tunnel junction based spin torque oscillators. Appl. Phys. Lett. 2009, 94, 112503. [CrossRef]

19. Zhou, Y.; Bonetti, S.; Persson, J.; Akerman, J. Capacitance enhaced synchronization of pairs of spin-transfer oscillators. IEEE Trans. Magn. 2009, 45, 2421-2423. [CrossRef]

20. Persson, J.; Zhou, Y.; Akerman, J. Phase-locked spin torque oscillators: Impact of device variablility and time delay. J. Appl. Phys. 2007, 101, 09A503. [CrossRef] 
21. Arbitrary Function Generators, AFG3000C Series Datasheet. Available online: https://www.newark.com/tektronix/afg3251ccal/cal-func-arbitrary-wf-generator/dp/12AC7990 (accessed on 30 August 2021).

22. Bonetti, S.; Muduli, P.; Mancoff, F.; Åkerman, J. Spin torque oscillator frequency versus magnetic field angle: The prospect of operation beyond 65 GHz. Appl. Phys. Lett. 2009, 94, 102507. [CrossRef]

23. Jhon, H.; Kim, M.; Kang, M. Cost-Effective 4 GHz VCO Using Only Miniature Spirals Realized in a $0.18 \mu \mathrm{m}$ CMOS Process for Wireless Sensor Network (WSN) Applications. Electronics 2019, 8, 1369. [CrossRef]

24. Hejazi, A.; Pu, Y.; Lee, K. A Design of Wide-Range and Low Phase Noise Linear Transconductance VCO with $193.76 \mathrm{dBc} / \mathrm{Hz}$ FoMT for mm-Wave 5G Transceivers. Electronics 2020, 9, 935. [CrossRef]

25. Alvero-Gonzalez, L.; Medina, V.; Kampus, V.; Paton, S.; Hernandez, L.; Gutierrez, E. Ring-Oscillator with Multiple Transconductors for Linear Analog-to-Digital Conversion. Electronics 2021, 10, 1408. [CrossRef] 